\title{
LETTER
}

\section{Lower tidal volumes in Brazil, also in patients without acute respiratory distress syndrome?}

\author{
Ary Serpa Neto, ${ }^{1,2, *}$ and Marcus J Schultz ${ }^{3,4}$ \\ See related research by Azevedo et al., http://ccforum.com/content/17/2/R63
}

In a recent study, Azevedo and colleagues [1] show tidal volumes $\left(\mathrm{V}_{\mathrm{T}}\right)$ to be low in patients in Brazilian ICUs. By showing this, it is clear that ventilation practice in Brazil mirrors worldwide changes, at least with regard to $V_{T}$ in patients with acute respiratory distress syndrome (ARDS) $[2,3]$.

Efforts to implement protective ventilation have been largely restricted to patients with ARDS, which is understandable since its beneficial effects were convincingly demonstrated in these patients only. It is not unreasonable, however, to consider that lower $\mathrm{V}_{\mathrm{T}}$ also benefits patients without ARDS [4], although it could also be argued that lower $V_{T}$ strategies could harm patients without ARDS since it may increase sedation and maybe even paralysis needs, which are associated with increased incidence of delirium, ventilator-induced diaphragm dysfunction, and longer duration of ventilation. Furthermore, the new ARDS definition categorizes patients as having mild, moderate and severe ARDS [5]. It has been questioned whether attempts to control $\mathrm{V}_{\mathrm{T}}$ should be restricted to patients with moderate or severe ARDS. Consequently, the ICU community remains reluctant to use lower $\mathrm{V}_{\mathrm{T}}$ in patients without ARDS and patients only having mild ARDS, and desires randomized controlled trial (RCT) evidence. When planning a RCT, one would like to know to what $V_{T}$ the 'lower' $V_{T}$ is to be compared.

We would like to know, therefore, what $\mathrm{V}_{\mathrm{T}}$ was used in patients without ARDS, in patients with mild ARDS, and in patients with moderate or severe ARDS in Brazilian ICUs?

\section{Authors' response}

Luciano Cesar Pontes Azevedo, Jorge Ibrain Figueira Salluh, Marcio Soares

We would like to thank Drs Serpa-Neto and Schultz for their interest in our manuscript. We agree with the authors that there is now considerable evidence demonstrating the benefits of lung protective ventilation including lower tidal volumes in patients with ARDS [3] and even for ventilated patients without injured lungs [4]. However, this evidence is not currently translated into daily practice and our study provides additional data suggesting the common use of 'high' tidal volumes for patients with acute respiratory failure and also for the subgroup of those with ARDS $[1,6]$. We report (Table 1) data of tidal volumes per predicted body weight in the first day of mechanical ventilation of patients without ARDS, patients with mild ARDS (the previous definition of acute lung injury) and patients with moderate/severe

\footnotetext{
*Correspondence: aryserpa@terra.com.br

2Department of Critical Care Medicine, Hospital Israelita Albert Einstein, São Paulo, Brazil

Full list of author information is available at the end of the article
}

ARDS (the previous ARDS definition before the Berlin definitions). Median tidal volumes in all subgroups were above $6 \mathrm{ml} / \mathrm{kg}$ predicted body weight, without statistical difference between subgroups (Kruskall-Wallis test). Reasons for the lack of adherence to this strategy may include concerns about adverse effects of low tidal volumes, such as hypercapnia and increased need for sedatives, insufficient knowledge of the lung protective ventilation protocols and underrecognition of ARDS [7]. In conclusion, we believe more efforts are needed to identify the gaps that result in an incomplete translation of evidence to practice in order to guarantee implementation of the lung protective ventilation strategies on a daily basis.

\section{Abbreviations \\ ARDS, acute respiratory distress syndrome; $R C T$, randomized controlled trial; $V_{T}$, tidal volume. \\ Competing interests \\ The authors declare that they have no competing interests. \\ Author details \\ 'Department of Critical Care Medicine, ABC Medical School, Santo André, São Paulo, Brazil. ${ }^{2}$ Department of Critical Care Medicine, Hospital Israelita Albert}


Table 1. Data on tidal volumes on the first day of ventilatory support for patients without and with ARDS

\begin{tabular}{lccc}
\hline Parameter & Without ARDS & Mild ARDS & Moderate/severe ARDS \\
\hline Tidal volume day $1(\mathrm{ml} / \mathrm{kg})$ & $7.3(6.1-8.5)$ & $8.1(6.4-9.5)$ \\
\hline
\end{tabular}

Data are expressed as median (P25-P75). ARDS, acute respiratory distress syndrome.

Einstein, São Paulo, Brazil. ${ }^{3}$ Department of Intensive Care Medicine, Academic Medical Center, Amsterdam, the Netherlands. ${ }^{4}$ Laboratory of Experimental Intensive Care and Anesthesiology (L.E.I.C.A), Academic Medical Center, Amsterdam, the Netherlands.

Published: 12 June 2013

\section{References}

1. Azevedo LC, Park M, Salluh Jl, Rea-Neto A, Souza-Dantas VC, Varaschin P, Oliveira MC, Tierno PF, Dal-Pizzol F, Silva UV, Knibel M, Nassar Jr AP, Alves RA, Ferreira JC, Teixeira C, Rezende V, Martinez A, Luciano PM, Schettino G, Soares M: Clinical outcomes of patients requiring ventilatory support in Brazilian intensive care units: a multicenter, prospective, cohort study. Crit Care 2013, 17:R63.

2. Esteban A, Anzueto A, Frutos F, Alía I, Brochard L, Stewart TE, Benito S, Epstein SK, Apezteguía C, Nightingale P, Arroliga AC, Tobin MJ; Mechanical Ventilation International Study Group: Characteristics and outcomes in adult patients receiving mechanical ventilation: a 28-day international study. JAMA 2002, 287:345-355.

3. Villar J, Blanco J, Añón JM, Santos-Bouza A, Blanch L, Ambrós A, Gandía F, Carriedo D, Mosteiro F, Basaldúa S, Fernández RL, Kacmarek RM; ALIEN Network: The ALIEN study: incidence and outcome of acute respiratory distress syndrome in the era of lung protective ventilation. Intensive Care Med 2011, 37:1932-1941.
4. Serpa Neto A, Cardoso SO, Manetta JA, Pereira VG, Esposito DC, Pasqualucci Mde O, Damasceno MC, Schultz MJ: Association between use of lungprotective ventilation with lower tidal volumes and clinical outcomes among patients without acute respiratory distress syndrome: a metaanalysis. JAMA 2012, 308:1651-1659.

5. ARDS Definition Task Force, Ranieri VM, Rubenfeld GD, Thompson BT, Ferguson ND, Caldwell E, Fan E, Camporota L, Slutsky AS: Acute respiratory distress syndrome: the Berlin Definition. JAMA 2012, 307:2526-2533.

6. Linko R, Okkonen M, Pettila V, Perttila J, Parviainen I, Ruokonen E, Tenhunen J, Ala-Kokko T, Varpula T: Acute respiratory failure in intensive care units. FINNALI: a prospective cohort study. Intensive Care Med 2009, 35:1352-1361.

7. Cooke CR, Kahn JM, Watkins TR, Hudson LD, Rubenfeld GD: Costeffectiveness of implementing low-tidal volume ventilation in patients with acute lung injury. Chest 2009, 136:79-88.

doi:10.1186/cc12721

Cite this article as: Neto AS, Schultz MJ: Lower tidal volumes in Brazil, also in patients without acute respiratory distress syndrome? Critical Care 2013, $17: 436$. 